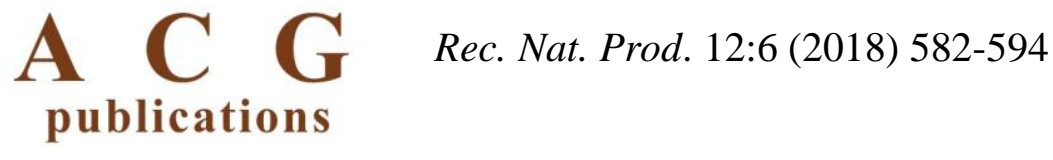

records of natural products

\title{
Mode of Action: Synergistic Interaction of Peppermint (Mentha $x$ piperita L. Carl) Essential Oil and Meropenem Against Plasmid-Mediated Resistant $E$. coli
}

\author{
Shun-Kai Yang $\odot^{1}$, Polly Soo-Xi Yap $\odot^{2}$, Thiba Krishnan ${ }^{\circledR}$, Khatijah \\ Yusoff $\odot^{4}$, Kok-Gan Chan $\odot^{4}$, Wai-Sum Yap $\odot^{5}$, Kok-Song Lai $\odot^{1}$ and \\ Swee-Hua Erin Lim ${ }^{6,7 *}$ \\ ${ }^{1}$ Department of Cell and Molecular Biology, Faculty of Biotechnology and Biomolecular Sciences, \\ Universiti Putra Malaysia, 43400 Serdang, Selangor, Malaysia \\ ${ }^{2}$ School of Postgraduate Studies and Research, International Medical University, No. 126, Jalan Jalil \\ Perkasa 19, Bukit Jalil, 57000 Kuala Lumpur, Malaysia \\ ${ }^{3}$ Division of Genetics and Molecular Biology, Institute of Biological Sciences, Faculty of Science, \\ University of Malaya, 50603 Kuala Lumpur, Malaysia \\ ${ }^{4}$ Department of Microbiology, Faculty of Biotechnology and Biomolecular Sciences, Universiti Putra \\ Malaysia, 43400 Serdang, Selangor, Malaysia \\ ${ }^{5}$ Department of Biotechnology, Faculty of Applied Sciences, UCSI University, 56000 Cheras, Kuala \\ Lumpur, Malaysia \\ ${ }^{6}$ Centre for Bioinformatics, School of Data Sciences, Perdana University, MAEPS Building, Serdang, \\ Selangor, Malaysia \\ ${ }^{7}$ Health Sciences Division, Abu Dhabi Women's College, Higher Colleges of Technology, 41012 Abu \\ Dhabi, United Arab Emirates
}

(Received December 22, 2017; Revised February 12, 2018; Accepted February 13, 2018)

\begin{abstract}
This study investigated the bactericidal mechanism of peppermint essential oil (PEO) when used singly and in combination with meropenem against multidrug resistant Escherichia coli. Chemical compositions of PEO were identified via GC-MS, followed by time-kill analysis which was performed to evaluate the antibacterial activities of PEO and meropenem. Furthermore, outer membrane permeability test, zeta potential measurement and scanning electron microscopy were performed to evaluate the ability of PEO in bacterial membrane disruption. Next, anti-quorum sensing assay was performed to assess the ability of PEO in quorum sensing inhibition. A complete killing activity was observed within five minutes of treatment with PEO and meropenem at sub-lethal concentrations. In addition, the outer membrane permeability test and zeta potential measurement performed indicated increase in the membrane permeability and membrane disruption which can be observed in the scanning electron micrograph. Furthermore, significant decrease in the light production of $E$. coli pSB1075 treated by PEO indicates the presence of quorum sensing inhibitors within PEO. The findings suggest that PEO has the ability to disrupt the bacterial outer membrane which increases membrane permeability, in addition to the possible inhibition of bacterial quorum sensing ability in multidrug resistant $E$. coli, aiding in the reversal of antibiotic resistance.
\end{abstract}

Keywords: Escherichia coli; essential oil; membrane permeability; Mentha x piperita L. Carl; quorum sensing; synergism. () 2018 ACG Publications. All rights reserved.

\footnotetext{
${ }^{*}$ Corresponding author: E-Mail: erinlim@ perdanauniversity.edu.my; Phone: +60389418646 ext. 178
}

The article was published by ACG Publications 


\section{Introduction}

Extensive use of antibiotics in hospitals, communities and agriculture have fueled the ever increasing threat of antibiotic resistant infections, fast tracking the evolution towards antibiotic resistance in microorganisms [1]. Escherichia coli and Klebsiella pneumonia expressing genes such as $C M Y, I M P, K P C, N D M, O X A$ and VIM which encode carbapenemases which have been described in numerous study and are surfacing at an alarming rate [2-5]. Gram negative bacteria often simultaneously activate two resistant mechanisms whereby the mass production of $\beta$-lactamases is coupled with the decrease in the permeability of their outer membrane (OM) [6-8], making a multitargeted drug therapy the ideal treatment method. Preferences for combination therapy by testing natural products alongside with existing antibiotics have been reported to exert synergistic effects [9]. However, limited information about the exact mechanisms of essential oil functioning as an antibiotic resistant modifier is known, despite countless reports demonstrating favorable synergistic interactions [10-13].

In the previous work by Yap et al. 2013, the minimum inhibitory concentration of both peppermint essential oil (PEO) $(8 \% \mathrm{v} / \mathrm{v})$ and meropenem $(4 \mu \mathrm{g} / \mathrm{mL})$ were determined via resazurin microplate assay [14]. In subsequent checkerboard assay, it was found that, when combined, PEO and meropenem reacted synergistically against KPC-3 producing E. coli pMG309, with significantly lowered minimum inhibitory concentration (MIC) (PEO, $1 \% \mathrm{v} / \mathrm{v}$; meropenem, $0.5 \mu \mathrm{g} / \mathrm{mL}$ ) [14]. This resulted in a fractional inhibitory concentration (FIC) index of 0.26 , indicating a high degree of synergism. The option for using essential oils in combination with antibiotics hold potential in offering possibly a novel, multi-targeted method in addressing the issue of antimicrobial resistance (AMR). A related study demonstrated that PEO and its major component menthol displayed synergistic activity with oxytetracycline. The finding postulated that the lowered antibiotic effective dosage needed to achieve total eradication of bacteria is attributed to the ability of PEO in plasmid curing [15]. However, the roles of the components within essential oils have not been explored thoroughly to date. Therefore, the objective of the current study was carried out to elucidate the possible mechanism of PEO when used against E. coli pMG309, which harbors plasmid encoding KPC-3 $\beta$-lactamase capable of hydrolyzing a wide range of $\beta$-lactam antibiotics such as, penicillins, cephalosporins and carbapenems [16]

\section{Materials and Methods}

\subsection{Peppermint (Mentha x piperita L. Carl) Essential Oil (PEO) and Meropenem}

The peppermint (Mentha x piperita L. Carl) essential oil (Serial number: 7212) purchased from Aroma Trading Ltd. (Milton Keynes, UK) was used throughout the studies. Both PEO and meropenem (Sigma Aldrich, St Louis, USA) were dissolved in Mueller-Hinton broth (MHB; oxoid, Cambrigde, UK) supplemented with Tween80, a solubilizing agent at $1 \%(\mathrm{v} / \mathrm{v})$ concentration to make desirable stock solution as described in the Clinical and Laboratory Standards Institute (CLSI) M100-S21 guidelines.

\subsection{Gas chromatography-mass Spectrometry (GC-MS) Analysis}

The GC-MS analysis was performed with Agilent GC-MS, 7890A GC System with a triple-axis detector (5975C MSD) and an HP-5MS column $(30 \mathrm{~m} \times 250 \mu \mathrm{m} \times 0.25 \mu \mathrm{m})$ (Agilent Technologies, California, USA). Helium was used as the carrier gas in the MS. The sample was injected with an auto-injector heated to $250{ }^{\circ} \mathrm{C}$ (Agilent Technologies 7693 Autosampler, California, USA). The oven column, on the other hand, was temperatureprogrammed from $40{ }^{\circ} \mathrm{C}(2 \mathrm{~min})$ to $175{ }^{\circ} \mathrm{C}$ at a rate of $5{ }^{\circ} \mathrm{C} \mathrm{min}^{-1}$ within $10 \mathrm{~min}$. The temperature then rose to $250{ }^{\circ} \mathrm{C}$ at a rate of $90{ }^{\circ} \mathrm{C} \mathrm{min}-1$ within the next $5 \mathrm{~min}$. The flow rate of the column was $1 \mathrm{~mL} \mathrm{~min}^{-1}$ with a split ratio of 40:1. EI mode with scan range $30-450 \mathrm{~m} / \mathrm{z}$ was used to analyze the MS. The temperature of the MS source was set at $250{ }^{\circ} \mathrm{C}$ whereas the MS quad at $150{ }^{\circ} \mathrm{C}$. Identification of compounds was solely based on the comparison of the 
mass spectra with those in National Institute of Standards and Technology libraries. The relative percentage of the identified compounds was computed from their GC peak area and only components with total abundance exceeding $0.1 \%$ were presented.

\subsection{Bacterial Strains and Growth Conditions}

Escherichia coli pMG309 harboring a plasmid encoding $\beta$-lactamase, KPC-3, a kind gift from George A. Jacoby (Lahey Clinic, MA, USA) was cultured in Mueller-Hinton broth (MHB; Oxoid, Cambridge, UK) and all test conditions were carried out according to the CLSI M07-A8 guidelines. Biosensor strains E. coli $p S B 1075$ and E. coli pSB401 used for the anti-quorum sensing assay were detailed in Table 1. Both biosensor strains were cultured in Luria Bertani (LB; Oxoid, Cambridge, UK) broth supplemented with the antibiotics tetracycline and ampicillin (Thermo Fisher Scientific, Massachusetts, USA) at $37{ }^{\circ} \mathrm{C}$ with shaking at $200 \mathrm{rpm}$.

Table 1. Biosensor E. coli strains and their plasmid used

\begin{tabular}{llc}
\hline Biosensor & Description & Source \\
\hline $\begin{array}{l}\text { Escherichia } \\
\text { coli } \text { pSB1075 }\end{array}$ & $\begin{array}{l}\text { lasR lasI' (Pseudomonas aeruginosa PAO1) : luxCDABE } \\
\text { (Photorhabdus luminescens ATCC 29999) fusion in pUC18 }\end{array}$ & [17] \\
& $\begin{array}{l}\text { AmpR, AHL biosensor producing bioluminescence in } \\
\text { response to long chain AHL. }\end{array}$ & \\
$\begin{array}{l}\text { Escherichia } \\
\text { coli pSB401 }\end{array}$ & $\begin{array}{l}\text { luxR luxI' (Photobacterium fischeri ATCC 7744) } \\
\text { fusion; } \text { pACYC184-derived, TetR, AHL biosensor producing } \\
\text { bioluminescence in response to short chain AHL. }\end{array}$ & \\
\hline
\end{tabular}

\subsection{Time-kill Assay}

The spread-plate method was used for viable counts of bacteria in the time kill assay. The sublethal concentrations of PEO and meropenem used in the time-kill assay were determined in previous work [14], control (without treatment); PEO (1\% v/v); meropenem $(0.5 \mu \mathrm{g} / \mathrm{mL}) ;$ PEO $(1 \% \mathrm{v} / \mathrm{v})$ in combination with meropenem $(0.5 \mu \mathrm{g} / \mathrm{mL})$. Directly after addition of the inoculum and after each interval of incubation (every $4 \mathrm{~h}$ ), $100 \mu \mathrm{L}$ of samples was removed for viable counting as a preliminary screening (data not shown). Sampling time was then shortened to 5, 10, 30, 60, 120, 180, $240 \mathrm{~min}$ in the events of rapid killing rate. Samples were serially diluted with $0.85 \%$ (w/v) sodium chloride, then plated onto Mueller-Hinton (MH) agar followed by an overnight incubation at $37^{\circ} \mathrm{C}$. The minimum time required to inhibit the growth of bacteria undergoing combinatory treatment was used in subsequent assays as the optimal treatment time. The experiment was performed in triplicates.

\subsection{Outer Membrane (OM) Permeability Test}

OM permeability test was performed using sub-lethal concentration of PEO (1\% v/v) and meropenem $(0.5 \mu \mathrm{g} / \mathrm{mL})$, as detailed previously [14], using $0.1 \%(\mathrm{w} / \mathrm{v})$ of anionic detergent, sodium dodecyl sulfate (SDS; Thermo Fisher Scientific, Massachusetts, USA) as a permeabilizing probe [18]. The absorbance at $625 \mathrm{~nm}$ were measured and compared amongst treatment group with and without SDS via a UV-visible and visible spectrophotometer (Thermo Fisher Scientific, Massachusetts, USA). The experiment was performed in 3 biological replicate in order to minimize the variance between sample of the same treatment group.

\subsection{Bacterial Surface Charge: Zeta Potential Measurement}

The zeta potential of all treatment groups was quantified using Nano Zetasizer (Malvern Instruments, Malvern, UK). After treatment with sub-lethal concentration of PEO (1\% v/v) and meropenem $(0.5 \mu \mathrm{g} / \mathrm{mL})$ alone, and in combination for 5 minutes, cells were washed thoroughly with 
phosphate buffered saline (PBS) (pH 7.4) for in preparation for measurements. The non-treated cell suspension in PBS was used as a control. The experiment was performed in 3 biological replicate in order to minimize the variance between sample of the same treatment group.

\subsection{Scanning Electron Microscopy}

SEM were performed on non-treated cells, cell treated with PEO (1\% v/v) alone and in combination with meropenem $(0.5 \mu \mathrm{g} / \mathrm{mL})$ for $5 \mathrm{~min}$, as determined in the time kill assay. After the 5 minute treatment, cells were harvested and washed with PBS ( $\mathrm{pH}$ 7.4) for at least three times to remove the treatment residue completely. Then, the samples were processed according to the reported protocol with slight modification [19]. Samples were fixed with $4 \%$ glutaraldehyde and $1 \%$ osmium tetroxide at $4{ }^{\circ} \mathrm{C}$ followed by dehydration via sequential exposure to graded acetone (35-100\%). The samples were then subjected to critical point drying and finally sputter-coated with gold, followed by SEM observations via JEOL JSM-6400 (JEOL Ltd., Tokyo, Japan). All treatment groups were compared visually to the untreated group based on the criteria of modification towards the cell surface (blebbing/corrugation) and cell to cell contact (adhesion).

\subsection{Anti-quorum Sensing Assay: Quantification of Light Production from E. coli pSB1075 and E. coli pSB401}

Light produced by both biosensor strains of E. coli was measured using an Infinite M200 Pro Tecan Microplate Reader (Tecan, Männedorf, Switzerland). A concentration of $0.5 \mu \mathrm{g} / \mathrm{mL} 3$-oxo-C6HSL and $10 \mu \mathrm{g} / \mathrm{mL}$ 3-oxo-C12-HSL (Sigma-Aldrich, Missouri, USA) were prepared in acetonitrile (Sigma-Aldrich, Missouri, USA) and stored at $-20^{\circ} \mathrm{C}$. An overnight culture of $E$. coli biosensor cells were diluted to $0.5 \mathrm{McFarland}$ Standard. Then, $200 \mu \mathrm{L}$ of $E$. coli biosensor cells with essential oil $(0.01 \%, 0.025 \%$ and $0.05 \%)$ was added into the well of Greiner 96 -well microtitre plate (Greiner BioOne, Kremsmünster, Austria). For comparison, E. coli pSB401 and E. coli pSB1075 were supplemented with $2 \mu \mathrm{L}$ of 3-oxo-C6-HSL $(0.005 \mu \mathrm{g} / \mathrm{mL})$ and $2 \mu \mathrm{L}$ of 3-oxo-C12-HSL $(0.1 \mu \mathrm{g} / \mathrm{mL})$, respectively. The light signal produced was determined every $30 \min$ for $24 \mathrm{~h}$ at $495 \mathrm{~nm}$ by the microplate reader. Production of light was measured as relative light units (RLU), whereby the total light production is directly proportional to the growth rate as well as the quorum sensing ability of the biosensor strains [20]. A decrease of light production in E. coli pSB1075 and E. coli pSB401 indicates anti-quorum sensing properties of the PEO.

\subsection{Statistical Analysis}

All results represent the average of three independent experiments. The data were presented as mean \pm standard deviation (SD) and analyzed by one-way analysis of variance (ANOVA) and Student's t-test. The $P$ value was calculated via GraphPad Prism 5 statistical software with $P<0.05$ considered as significant.

\section{Results and Discussion}

The chemical composition of peppermint (Piperita $x$ mentha) essential oil is tabulated in Table 2. The major compounds $(>1.0 \%)$ found in PEO are menthol $(50.1435 \%)$, followed by menthone (24.4276\%), menthyl acetate (6.1701\%), eucalyptol (5.7894\%), cis- $\beta$-Terpineol (1.8929\%), o-cymene (1.0384\%) and caryophyllene oxide (1.0148\%). 
Table 2. Chemical composition of peppermint (Mentha x piperita) essential oil

\begin{tabular}{|c|c|c|c|c|}
\hline Peak & Library/ID & $\mathrm{RI}^{\mathrm{a}}$ & $\mathrm{RI}^{\mathrm{b}}$ & Composition \% \\
\hline 1 & Menthol & 1177 & 1177 & 41.36 \\
\hline 2 & $p$-menthone & 1156 & 1150 & 25.72 \\
\hline 3 & $l$-menthone & 1166 & 1150 & 17.78 \\
\hline 4 & Isomenthol acetate & 1295 & 1295 & 4.59 \\
\hline 5 & Isopulegol & 1147 & 1145 & 1.60 \\
\hline 6 & Piperitone & 1256 & 1253 & 1.49 \\
\hline 7 & Caryophyllene & 1423 & 1420 & 1.15 \\
\hline 8 & $( \pm)$-pulegone & 1241 & 1234 & 1.07 \\
\hline 9 & Neoisomenthol & 1185 & 1173 & 0.96 \\
\hline 10 & Terpineol & 1193 & 1189 & 0.52 \\
\hline 11 & (-)- $\beta$-bourbonene & 1388 & 1384 & 0.46 \\
\hline 12 & (-)-lavandulol & 1169 & 1168 & 0.34 \\
\hline 13 & $p$-methan-1-ol & 1191 & 1110 & 0.25 \\
\hline 14 & Germacrene D & 1458 & 1480 & 0.22 \\
\hline 15 & Elixene & 1339 & 1337 & 0.19 \\
\hline 16 & D-limonene & 1028 & 1029 & 0.17 \\
\hline 17 & $\gamma$-muurolene & 1502 & 1476 & 0.14 \\
\hline 18 & Caryophyllene oxide & 1589 & 1580 & 0.13 \\
\hline 19 & Linalool & 1101 & 1099 & 0.12 \\
\hline 20 & (-)-elemene & 1395 & 1390 & 0.10 \\
\hline 21 & 1-decanol & 1274 & 1272 & 0.05 \\
\hline
\end{tabular}

Time-kill assay indicated synergism between PEO and meropenem whereby the combination treatment results in a reduction of $\geq 2-\log _{10} \mathrm{CFU} / \mathrm{mL}$ compared to the growth curve of cells treated singly with essential oil and antibiotic and the control [20]. The PEO and meropenem combination showed rapid and complete killing profile within 5 mins after the addition of PEO and meropenem. However, when applied singly, PEO or meropenem did not eradicate the cells completely or as rapidly as the combinatory counterpart (Figure 1).

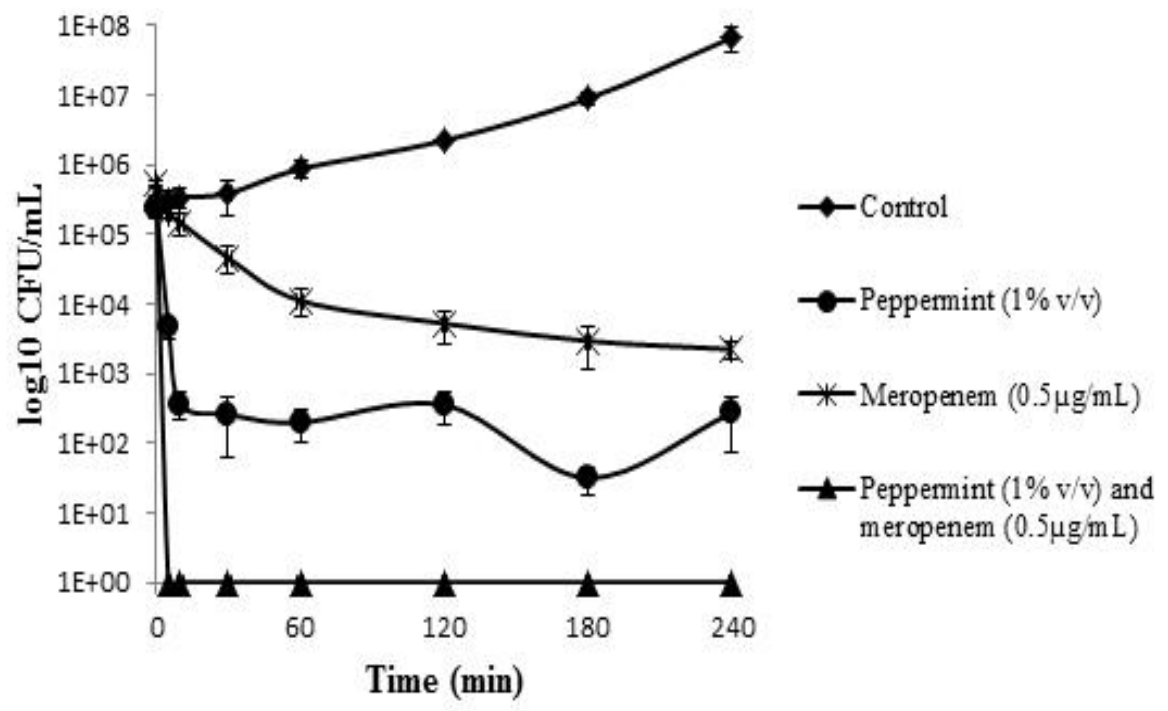

Figure 1. Growth curves of E. coli pMG309

Untreated E. coli pMG309 (४), E. coli pMG309 treated with PEO $(\bullet)$ and meropenem $(*)$ alone and in combination $(\boldsymbol{\Lambda})$. 
Sodium dodecyl sulfate (SDS) was the permeabilizing probe used to detect a disruption in the bacterial outer membrane in this experiment. Differences in absorbance between pre- and posttreatment of the control, essential oil or/and meropenem with or without $0.1 \%$ SDS was presented in Table 3. Sample treated with PEO alone and the combination of PEO and meropenem reduced the cell viability significantly $(P<0.05)$ when compared to the non-treated group after 5 minutes of treatment time (denoted by superscript a in Table 3). Absorbance of cells treated with meropenem alone (with and without $0.1 \% \mathrm{SDS})$ remained constant throughout the study and was not significant $(P>0.05)$.

Table 3. Outer membrane permeabilization of E. coli pMG309 treated with PEO and/or meropenem

\begin{tabular}{|c|c|c|c|c|c|}
\hline \multirow{2}{*}{$\begin{array}{l}\text { Treatment } \\
\text { Time (min) }\end{array}$} & \multicolumn{5}{|c|}{$\mathrm{OD}_{625} \pm \mathrm{SD}(\mathrm{n}=3)$} \\
\hline & 0 & 5 & 10 & 30 & 60 \\
\hline \multicolumn{6}{|l|}{ Control } \\
\hline with $0.1 \%$ SDS & $0.33 \pm 0.001$ & $0.32 \pm 0.005$ & $0.31 \pm 0.006$ & $0.30 \pm 0.001$ & $0.30 \pm 0.002$ \\
\hline without $0.1 \%$ SDS & $0.32 \pm 0.003$ & $0.33 \pm 0.003$ & $0.33 \pm 0.004$ & $0.33 \pm 0.005$ & $0.33 \pm 0.006$ \\
\hline \multicolumn{6}{|l|}{ Peppermint $(1 \% \mathrm{v} / \mathrm{v})$} \\
\hline with $0.1 \%$ SDS & $0.25 \pm 0.005^{\mathrm{a}}$ & $0.23 \pm 0.007^{\mathrm{b}}$ & $0.22 \pm 0.007^{b}$ & $0.21 \pm 0.001^{\mathrm{b}}$ & $0.22 \pm 0.003^{b}$ \\
\hline without $0.1 \%$ SDS & $0.26 \pm 0.001^{\mathrm{a}}$ & $0.26 \pm 0.004^{\mathrm{b}}$ & $0.25 \pm 0.002^{\mathrm{b}}$ & $0.25 \pm 0.003^{\mathrm{b}}$ & $0.26 \pm 0.011^{\mathrm{b}}$ \\
\hline \multicolumn{6}{|c|}{ Meropenem $(0.5 \mu \mathrm{g} / \mathrm{mL})$} \\
\hline with $0.1 \%$ SDS & $0.33 \pm 0.002$ & $0.33 \pm 0.005$ & $0.32 \pm 0.007$ & $0.33 \pm 0.010$ & $0.33 \pm 0.003$ \\
\hline without $0.1 \%$ SDS & $0.33 \pm 0.002$ & $0.32 \pm 0.010$ & $0.33 \pm 0.009$ & $0.32 \pm 0.001$ & $0.32 \pm 0.005$ \\
\hline \multicolumn{6}{|c|}{ Peppermint $(1 \% \mathrm{v} / \mathrm{v})+$ Meropenem $(0.5 \mu \mathrm{g} / \mathrm{mL})$} \\
\hline with $0.1 \%$ SDS & $0.26 \pm 0.004^{\mathrm{a}}$ & $0.23 \pm 0.006^{\mathrm{b}}$ & $0.21 \pm 0.002^{\mathrm{b}}$ & $0.21 \pm 0.006^{\mathrm{b}}$ & $0.20 \pm 0.008^{b}$ \\
\hline without $0.1 \%$ SDS & $0.26 \pm 0.004^{\mathrm{a}}$ & $0.25 \pm 0.004^{\mathrm{b}}$ & $0.25 \pm 0.005^{\mathrm{b}}$ & $0.26 \pm 0.004^{\mathrm{b}}$ & $0.25 \pm 0.003^{b}$ \\
\hline
\end{tabular}

${ }^{a}$ Significant difference among treatment groups when compared to the corresponding control groups (with or without $0.1 \%$ SDS $).(P<0.05)$

${ }^{\mathrm{b}}$ Significant difference between samples treated and non-treated with $0.1 \%$ SDS at the corresponding time points. $(P<0.05)$

Values are mean $\mathrm{OD}_{625} \pm \mathrm{S}$.D of three replicates.

The surface charge of bacterial cells is determined based on their zeta potential value, which can be measured based on the mobility of cells exposed to an electrophoretic force under defined $\mathrm{pH}$ and salt concentrations. Under normal condition, E. coli pMG309 had an original negative surface charge of $-11.52 \mathrm{mV}$. Singular treatment of PEO and meropenem further increases the surface charge to $-2.82 \mathrm{mV}$ and $-3.67 \mathrm{mV}$ while combinatory treatment yielded the further increases the surface charge to -2.56 . Thus, all the treatments applied significantly reduced the negative charges on the cell surface with the cells treated with the combination of PEO and meropenem being the least negative ( $P$ $<0.05$ ) (Figure 2). 


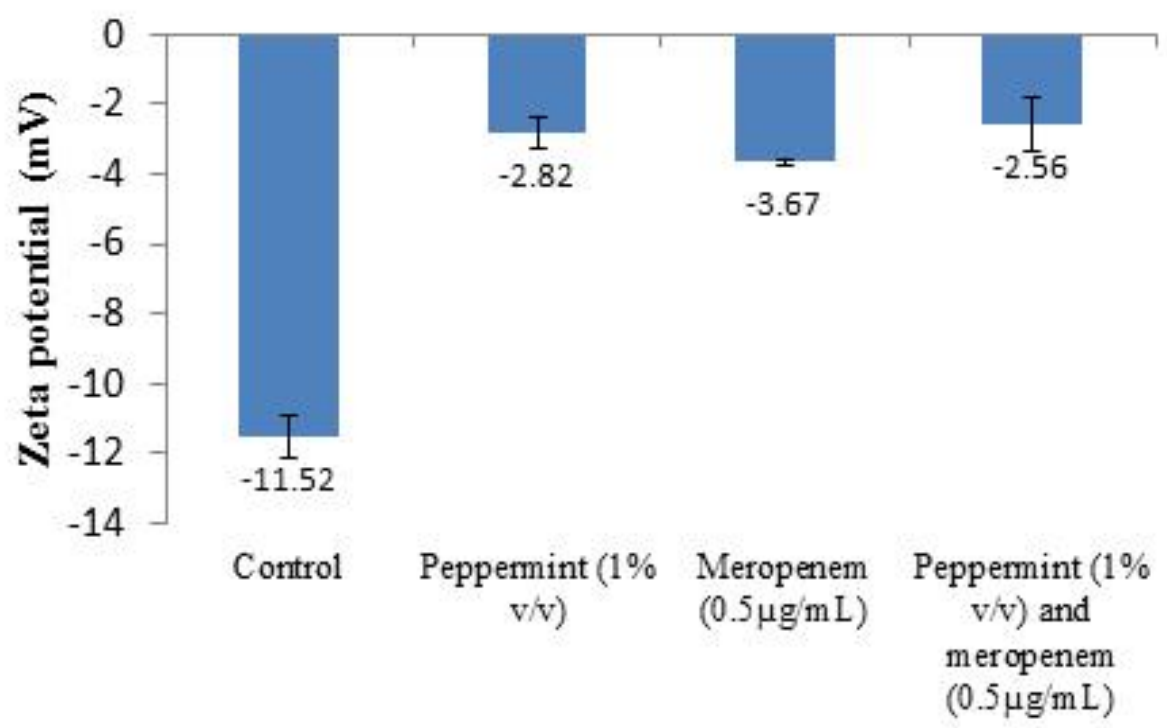

\section{Samples}

Figure 2. Zeta potential values $(\mathrm{mV})$ of E. coli pMG309 when treated with PEO or/and meropenem
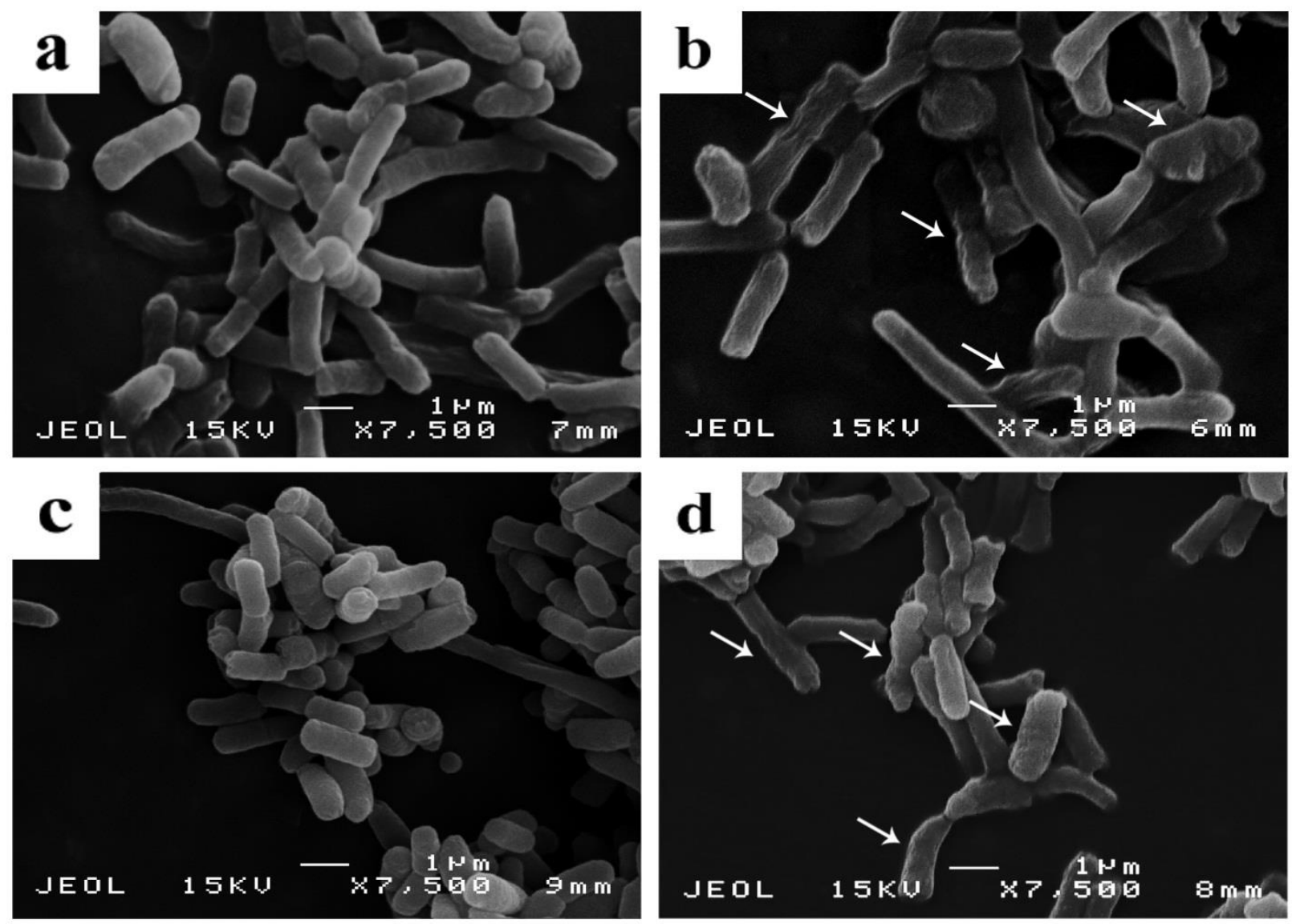

Figure 3. Scanning electron micrographs of E. coli pMG309

(a) Untreated E. coli pMG309, (b) cells treated with PEO (1\% v/v), (c) cells treated with meropenem $(0.5 \mu \mathrm{g} / \mathrm{mL})$ and (d) cells treated with the combination of peppermint $(1 \% \mathrm{v} / \mathrm{v})$ and meropenem $(0.5 \mu \mathrm{g} / \mathrm{mL})$ for five minutes. The outer membrane of the treated cells appeared to be corrugated (shown in white arrows). 
The scanning electron micrographs further depict the morphological changes of $E$. coli pMG309 treated with PEO and meropenem, alone and in combination (Figure 3). In the non-treated controls, a rod-shaped morphology which is characteristic of E. coli was observed (Figure 3a). It was observed that samples treated with PEO alone (Figure 3b) and in combination with meropenem (Figure 3d) resulted in severe corrugation of the bacterial cell membrane when compared with untreated sample (Figure 3a) and sample treated with meropenem alone (Figure 3c).

An anti-quorum sensing assay was also performed to investigate the ability of PEO as a quorum sensing inhibitor by using biosensor E. coli. In the presence of a quorum sensing inhibitor, light production by the biosensor cells will decrease. Prior to the assay, resazurin microplate assay were used to determine the concentration of PEO that can be used for the anti-QS assay without harming the biosensor cell (data not shown). As observed from Figure 4, increment in the concentration of PEO from $0.01 \%, 0.025 \%$ to $0.05 \%$ significantly inhibited light production activity in E. coli pSB1075 (Figure 4a) but not in E. coli pSB401 (Figure 4b) when compared to the controls (untreated cells and cells treated with Tween80).
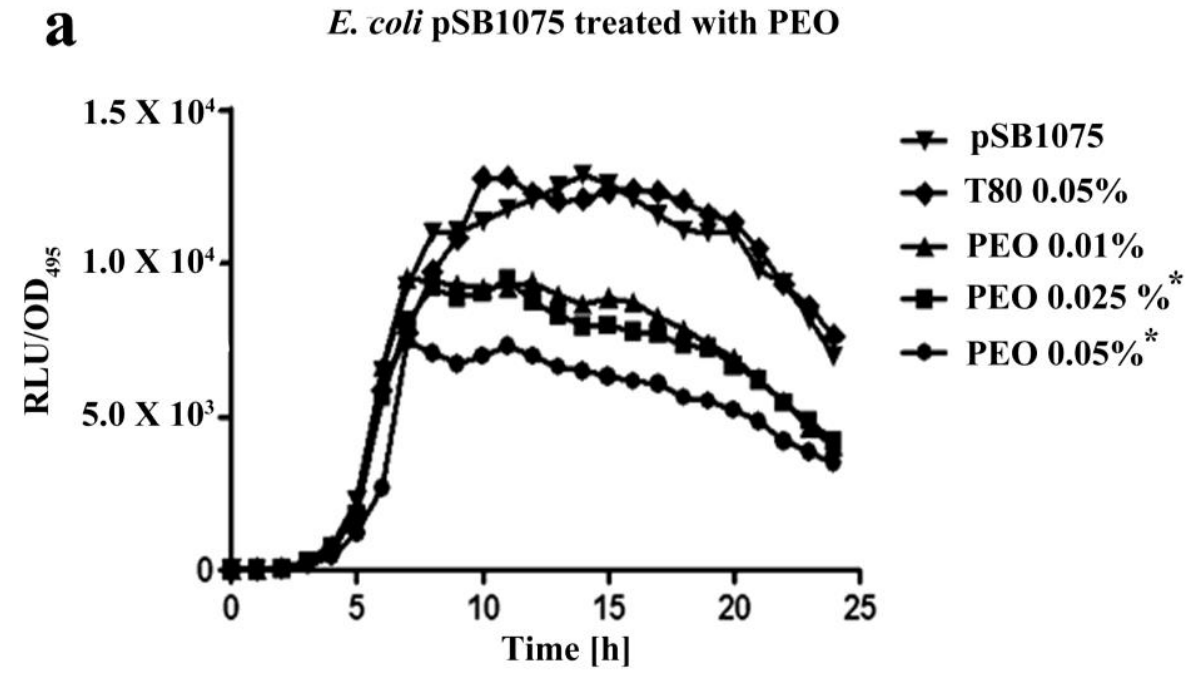

b E. coli pSB401 treated with PEO

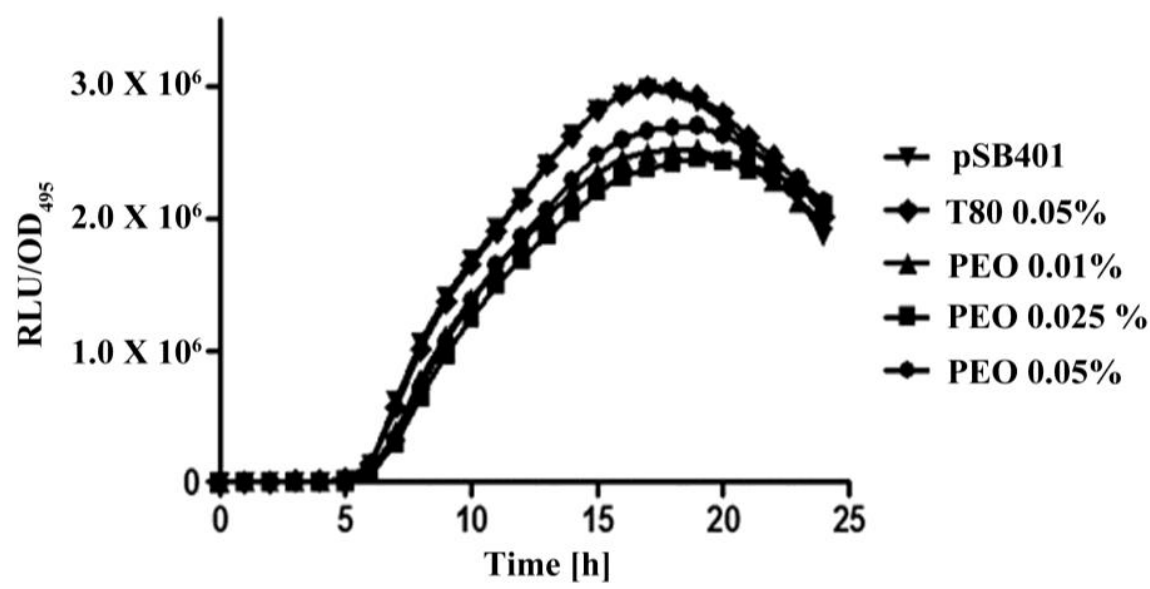

Figure 4. Light production by biosensor E. coli $\mathrm{pSB} 1075$ and E. coli $\mathrm{pSB} 401$

Both biosensor cells were treated with PEO at concentration $0.01 \%(\boldsymbol{\Delta}), 0.025 \%(\boldsymbol{\bullet})$, and $0.05 \%(\bullet)$. Tween 80 (४) and E. coli pSB401 and pSB1075 supplemented with 3-oxo-C6-HSL and 3-oxo-C12-HSL, were also included respectively, serving as controls $(\boldsymbol{\nabla})$. The data were presented as RLU/OD to account for any differences in growth. Data were analyzed via one-way analysis of variance, with $p<0.05$ being significant (indicated by $*$ in figure). 
In previous publication [14], five essential oils (cinnamon bark, tea tree, peppermint, marjoram and lavender essential oil) and seven antibiotics (ampicillin, carbenicillin, cefazolin, cefuroxime, piperacillin, ceftazidime and meropenem) had been screened for their minimum inhibitory concentration individually against 3 different strains of $E$. coli harboring different plasmid which encodes different $\beta$-lactamases, via resazurin microplate assay [14]. Following that, checkerboard assay had also been performed on all possible combinations of essential oil and antibiotics. Of all pairs, only 4 combinations of EO and antibiotic exhibited synergism with one another in nature and one of it was the combination of PEO and meropenem against $E$. coli pMG309 harboring KPC-3 $\beta$ lactamase. The MIC of PEO and meropenem were $8 \%$ and $4 \mu \mathrm{g} / \mathrm{mL}$ respectively when used alone. However, when applied in combination, the MIC of PEO and meropenem were reduced to $1 \%$ and 0.5 $\mu \mathrm{g} / \mathrm{mL}$ respectively, yielding a high degree of synergism with fractional inhibitory concentration index of 0.26 . Thus, this particular combination was chosen to be further studied in the current work.

GC-MS had been performed in order to understand and postulate the possible compounds which might be associated with the mechanism of PEO in combinatory treatment. According to the GC-MS analysis, PEO consisted of a total of 21 compounds $(\geq 0.1 \%)$ as presented in Table 2 . The major compounds found within PEO were menthol (41.36 \%), p-menthone $(25.72 \%), l$-menthone (17.78\%) and isomenthol acetate (4.59\%). For comparison, a previous study in Poland reported that menthol, menthone, and menthyl acetate were the major components in PEO [22]. In addition, similar studies carried out by researchers in Germany and Africa also obtained a similar result whereby menthol and menthone are the major components of PEO [23, 24]. According to the study conducted by [24], it was found that menthol disturbs the plasma membrane of bacteria, resulting in the alteration of membrane permeability and intracellular leakage [25]. However, there is no reported mechanism to date regarding the second most abundant component in PEO, menthone.

In the time-kill analysis, combinatory treatment with PEO and meropenem at their sub-lethal concentrations showed a complete and rapid killing profile when compared to the other three treatment groups. Therefore the bactericidal activity of PEO was not affected by the $\beta$-lactamase produced. Action of PEO against the E. coli might be due to the general toxicity effects of the essential oil itself on the bacterial outer membrane [25]. A similar study carried out on one of the major components of peppermint, menthol also caused disruption of outer membrane, leading to an increase in the membrane permeability and leakage of intracellular content [26]. To further validate this theory, the OM permeability test and zeta potential measurement were performed to assess the ability of PEOs in altering the OM permeability. In OM permeability test, anionic detergent, SDS was used as a permeabilizing probe whereby it was excluded extracellularly by the OM under normal condition [27]. However, a disruption in the membrane enabled the influx of SDS which eventually led to cell death when the accumulated concentration of SDS reached its threshold. Thus, SDS functions as a permeabilizing probe whereby it initiated cell lysis after the bacterial membrane had reached a critical extent of membrane disordering [28,29]. As shown in Table 3, PEO alone and PEO-meropenem combination altered the OM permeability of E. coli pMG309 significantly when compared to the other two treatment groups. Sub-lethal concentration of meropenem alone did not increase outer membrane permeability, thus there were no influx of SDS into the cells observed wherein the OD readings were not decreased significantly $(P>0.05)$ throughout the entire course of this experiment. This is due to the hydrophilic nature of meropenem, a $\beta$-lactam antibiotic which did not cause any lipopolysaccharide destabilisation, and thus did not potentiate the influx of SDS into the cells [29]. These results suggest that the outer membrane was disrupted solely by PEO, increasing the bacterial membrane permeability, subsequently decreasing the membrane's ability to osmoregulate the cell adequately, thus, promoting the influx of SDS, eventually lysing the cell [30]. In the current study, the possible mode of action of PEO is through the disruption of the bacterial membrane, either directly or indirectly, both at lethal and sub-lethal concentrations which increases the non-specific penetration of the antibiotic into the bacteria [31,32]. As discussed before [33], alteration in the OM of the bacteria would facilitate the diffusion of antibiotics through the cell barrier eventually elevating the intracellular concentration of antibiotics which subsequently leads to cell death [33]. This explains the synergism observed which caused rapid killing of multidrug resistant E. coli as observed in the timekill assay. Zeta potential measurement was then carried out to further clarify the effect of PEOs on the bacterial membrane. The zeta potential of bacterial cell membrane provides information of the membrane potential which reflects the metabolic state of the bacteria, whereby the zeta potential 
values were inversely proportional to the growth rate of the bacteria $[34,35]$. Bacterial cell surfaces are normally negatively charged under physiological conditions due to the presence of anionic groups such as carboxyl and phosphate in their membranes but the value becomes less negative when the bacteria is under stressful conditions. In this study, the zeta potential of E. coli pMG309 treated with PEO alone and in combination with meropenem was significantly higher than the non-treated cells, indicating disruption in the membrane thus leading to slow metabolic rate. This might be due to the acidification of $\mathrm{OM}$ by the PEO which increases the zeta potential of the bacteria, leading to membrane damage [36].

The scanning electron micrograph (Figure 3) revealed that the overall cell surface of E. coli pMG309 treated with PEO alone and in combination with meropenem were structurally different from the control, validating the ability of PEO in bacterial OM disruption. Observed morphological changes were due to the disruption of outer membrane as evidenced by the OM permeability test and zeta potential measurement as well as the previous findings by other researchers [37-39]. As shown in Figure 3c, cells treated with meropenem alone had minor corrugation on their outer membrane. Similar observations have been reported on bacterial cells treated with imipenem [40]. The minor corrugation was attributed to the nature of $\beta$-lactam antibiotic which interferes with the peptidoglycan linkage, eventually altering the integrity of the cell towards osmotic pressure and thus disrupting the outer membrane [41]. As shown in Figure $3 \mathrm{~b}$ and $3 \mathrm{~d}$, a severe alteration on the cellular surface of $E$. coli pMG309 was observed in the presence of PEO alone and in combination with meropenem. Therefore, OM permeability test, zeta potential measurement and scanning electron microscopy used in this study has provided strong evidence that PEO possess the capability to disrupt the outer membrane of E. coli pMG309, leading to an influx of meropenem into the cell which eventually eradicates the cell synergistically.

In this study, the anti-quorum sensing assay had also been carried out to detect the quorum sensing inhibitor compound which might be present within PEO. Quorum sensing is the language used between bacteria to communicate with each other solely through chemical signalling. QS regulates a vast number of bacterial functions including virulence and pathogenic potential of bacteria [41]. This communicative capability is also one of the major causes of antibiotic resistance nowadays. Under the stress of antimicrobials, a bacterial community produces signals which enhance the secretion of extracellular polymeric substances, leading to the formation of biofilm which serves as a protective shield to the bacterial community from antibiotics [42]. Meropenem is a broad-spectrum carbapenem antibiotic with a known mode of action that penetrates bacterial cells readily and interferes with the synthesis of the vital cell wall components [43]. In this current study, PEO was tested individually to investigate its anti-quorum sensing potential as an important anti-infective target that does not rely on antibiotics. Hence, the combination of meropenem/PEO aims to address gaps in the development carbapenem resistance such as increased expression of the efflux system, porin deficiency and increased chromosomal carbapenemases activity [44]. Results shown in Figure 4 indicates that increment in the concentration of peppermint oil significantly decreases the light production in biosensor $E$. coli pSB1075, subsequently increasing in quorum sensing inhibition. However, quorum sensing inhibition was not observed in control biosensor E. coli pSB401. Hence, PEO was shown to inhibit quorum sensing activity in $E$. coli pSB1075 carrying the las $R$ receptor gene but not in $E$. coli pSB401 carrying the luxR. las $R$ gene is commonly found in opportunistic pathongens such as $P$. aeruginosa while luxR gene is found in harmless bacteria such as Vibrio harveyi [45].

One of the most prominent mechanisms postulated in recent studies for the synergistic interactions between essential oil and antibiotics is via the disruption of the bacterial cytoplasmic membrane. Further investigation by researchers also showed the specific mechanism of essential oil components responsible for the leakage of potassium ions, loss of 260-nm-absorbing material and salt tolerance as well as up/down-regulation of protein found in bacterial membrane $[37,38,40,46]$. A standardized method for the evaluation of membrane permeability is yet to be developed and limitations exist whereby the activity of the compounds tested in unrelated studies cannot be compared to each other directly.

In the current study, PEO was found to act synergistically with meropenem showing disruption of the bacterial membrane as evidenced by findings from the OM permeability test, zeta potential measurement and scanning electron microscopy (SEM) observation. The findings implied that PEO has the potential to increase the bacterial susceptibility towards $\beta$-lactam antibiotics; in this 
case, meropenem may operate via two mechanisms: generalized bacterial outer membrane disruption which potentiates antibiotics influx into the cytoplasm, eventually leading to cell death and antiquorum sensing ability. It should be pointed out that this study serves as preliminary work on the mode of action of PEO in its synergistic interaction with meropenem. It has been discussed and hypothesized that the actions of PEO are broad and may provide promising cell targets other than the membrane effects. This possibility deserves further investigation. Additionally, since the membrane permeabilizing effects of PEO are demonstrated, their possible effect on up/down-regulation of the membrane proteins opens an interesting avenue for further study using high-throughput screening such as microarray. Further work such as better understanding of the mechanisms at the molecular level are required before the actual applications of combinatory therapy using essential oils and antibiotics as a potential drug treatment strategy in the clinical setting can be fully employed.

\section{Acknowledgments}

This study was funded by the Fundamental Research Grant Scheme (FRGS) from the Ministry of Higher Education (MOHE), Malaysia [FRGS/1/2011/SKK/IMU/03/3] and UCSI University Internal Grant [Proj-In-FAS-044]. The author (Kok Gan Chan) acknowledges Universiti Malaya for the High Impact Research Grant [HIR/MOHE Grant A000001-50001]. Authors would also like to thank Dr. George A Jacoby for providing the bacterial strain used in this study and fellow members of IMU Research Laboratory and Floral Biotechnology Laboratory, Universiti Putra Malaysia. SKY and PSXY drafted the manuscript. KSL and KY edited the manuscript whereas SHEL significantly refined the manuscript. All authors read and approved the final manuscript.

\section{ORCID}

Shun Kai Yang: 0000-0002-9275-3653

Polly Soo Xi Yap: 0000-0002-1615-3138

Thiba Krishnan: 0000-0003-1791-7627

Khatijah Yusoff: $0000-0002-7683-4760$

Kok Gan Chan: 0000-0002-1883-1115

Wai Sum Yap: 0000-0003-1589-0176

Kok Song Lai: 0000-0002-1887-2232

Swee Hua Erin Lim: 0000-0001-5177-0257

\section{References}

[1] S.K. Yang, L.Y. Low, P.S.X. Yap, K. Yusoff, C.W. Mai, K.S. Lai and S.H.E. Lim (2018). Plant-derived antimicrobials: insights into mitigation of antimicrobial resistance, Rec. Nat. Prod. 12(4), 295-316.

[2] P. Nordmann, G. Cuzon and T. Naas (2009). The real threat of Klebsiella pneumoniae carbapenemaseproducing bacteria, The Lancet Infect. Dis. 9(4), 228-236.

[3] W.H. Goessens, A.K. van der Bij, R. van Boxtel, J.D. Pitout, P. van Ulsen and D.C. Melles (2013). Antibiotic trapping by plasmid-encoded CMY-2 beta-lactamase combined with reduced outer membrane permeability as a mechanism of carbapenem resistance in Escherichia coli, Antimicrob. Agents. Chemother. 57(8), 3941-3949.

[4] H. Yigit, A.M. Queenan, G.J. Anderson, A. Domenech-Sanchez, J.W. Biddle and C.D. Steward (2001). Novel carbapenem-hydrolyzing beta-lactamase, KPC-1, from a carbapenem-resistant strain of Klebsiella pneumoniae, Antimicrob. Agents Chemother. 45(4), 1151-1161.

[5] D. Yong, M.A. Toleman, C.G. Giske, H.S. Cho, K. Sundman and K. Lee (2009). Characterization of a new metallo-beta-lactamase gene, blaNDM-1, and a novel erythromycin esterase gene carried on a unique genetic structure in Klebsiella pneumoniae sequence type 14 from India, Antimicrob. Agents. Chemother. 53(12), 5046-5054.

[6] M. Doumith, M.J. Ellington, D.M. Livermore and N. Woodford (2009). Molecular mechanisms disrupting porin expression in ertapenem-resistant Klebsiella and Enterobacter spp. clinical isolates from the UK, $J$. Antimicrob. Chemother. 63(4), 659-667. 
[7] Y.F. Liu, J.J. Yan, W.C. Ko, S.H. Tsai and J.J. Wu (2008). Characterization of carbapenem-non-susceptible Escherichia coli isolates from a university hospital in Taiwan, J. Antimicrob. Chemother. 61(5), 10201023.

[8] Q. Yang, H. Wang, H. Sun, H. Chen, Y. Xu and M. Chen (2010). Phenotypic and genotypic characterization of Enterobacteriaceae with decreased susceptibility to carbapenems: results from large hospital-based surveillance studies in China, Antimicrob. Agents Chemother. 54(1), 573-577.

[9] W.T. Langeveld, E.J. Veldhuizen and S.A. Burt (2013). Synergy between essential oil components and antibiotics: a review, Crit. Rev. Microbiol. 40(1), 76-94.

[10] H. Si, J. Hu, Z. Liu and Z. Zeng (2008). Antibacterial effect of oregano essential oil alone and in combination with antibiotics against extended-spectrum b-lactamase-producing Escherichia coli, FEMS Immunol. Med. Microbiol. 53, 190-194.

[11] A. Rosato, C. Vitali, N. De Laurentis, D. Armenise and M. Antonietta Milillo (2007). Antibacterial effect of some essential oils administered alone or in combination with Norfloxacin, Phytomedicine 14(11), 727732.

[12] N. Radulovic, M. Misic, J. Aleksic, D. Dokovic, R. Palic and G. Stojanovic (2007). Antimicrobial synergism and antagonism of salicylaldehyde in Filipendula vulgaris essential oil, Fitoterapia 78(7-8), 565-570.

[13] K. Palaniappan and R.A. Holley (2010). Use of natural antimicrobials to increase antibiotic susceptibility of drug resistant bacteria, Int. J. Food Microbiol. 140(2-3), 164-168.

[14] P.S. Yap, S.H. Lim, C.P. Hu and B.C. Yiap (2013). Combination of essential oils and antibiotics reduce antibiotic resistance in plasmid-conferred multidrug resistant bacteria, Phytomedicine 20(8-9), 710-713.

[15] G. Spengler, A. Molnar, Z. Schelz, L. Amaral, D. Sharples and J. Molnar (2006). The mechanism of plasmid curing in bacteria, Curr. Drug Target. 7(7), 823-841.

[16] J. Alba, Y. Ishii, K. Thomson, E.S. Moland and K. Yamaguchi (2005). Kinetics study of KPC-3, a plasmidencoded class A carbapenem-hydrolyzing beta-lactamase, Antimicrob. Agents Chemother. 49(11), 47604762.

[17] M.K. Winson, S. Swift, L. Fish, J.P. Throup, F. Jorgensen, S.R. Chhabra, B.W. Bycroft, P. Williams and G.S.A.B. Stewart (1998). Construction and analysis of luxCDABE-based plasmid sensors for investigating $\mathrm{N}$-acyl homoserine lactone-mediated quorum sensing, FEMS. Microbiol. Lett. 163(2), 185-192.

[18] P.S. Yap, T. Krishnan, K.G. Chan and S.H. Lim (2015). Antibacterial mode of action of Cinnamomum verum bark essential oil, alone and in combination with piperacillin, against a multi-drug-resistant Escherichia coli strain, J. Microbiol. Biotechnol. 25(8), 1299-1306.

[19] K.S. Lai, K. Yusoff and M. Maziah (2011). Extracellular matrix as the early structural marker for Centella asiatica embryogenic tissues, Biol. Plant. 55, 549-553.

[20] K. Winzer, C. Falconer, N.C. Garber, S.P. Diggle, M. Camara and P. Williams (2000). The Pseudomonas aeruginosa lectins PA-IL and PA-IIL are controlled by quorum sensing and by RpoS, J. Bacteriol. 182(22), 6401-6411.

[21] V. Lorian (2005). Antibiotics in laboratory medicine, 5th edition. Lippincott Williams \& Wilkins, Philadelphia.

[22] A. Bertoli, M. Leonardi, J. Krzyzanowska, W. Oleszek and L. Pistelli (2012). In vitro production of $M$. $x$ piperita not containing pulegone and menthofuran, Acta Biochim. Pol. 59(3), 417-423.

[23] I.H. Bassole, A. Lamien-Meda, B. Bayala, S. Tirogo, C. Franz and J. Novak (2010). Composition and antimicrobial activities of Lippia multiflora Moldenke, Mentha x piperita L. and Ocimum basilicum L. essential oils and their major monoterpene alcohols alone and in combination, Molecules 15(11), 7825 7839.

[24] E. Schmidt, S. Bail, G. Buchbauer, I. Stoilova, T. Atanasova and A. Stoyanova (2009). Chemical composition, olfactory evaluation and antioxidant effects of essential oil from Mentha x piperita, Nat. Prod. Commun. 4(8), 1107-1112.

[25] D.G. Barceloux (2008). Peppermint Oil (Mentha $x$ piperita L.), in medical toxicology of natural substances: foods, fungi, medicinal herbs, plants, and venomous animals. John Wiley \& Sons, Inc, New Jersey.

[26] D. Trombetta, F. Castelli, M.G. Sarpietro, V. Venuti, M. Cristani and C. Daniele (2005). Mechanisms of antibacterial action of three monoterpenes, Antimicrob. Agents Chemother. 49(6), 2474-2478.

[27] S.K. Yang, K. Yusoff, C.W. Mai, W.M. Lim, W.S. Yap, S.H.E. Lim and K.S. Lai (2017). Additivity vs synergism: Investigation of the additive interaction of cinnamon bark oil and meropenem in combinatory therapy, Molecules 22(11), 1733.

[28] M. Nazari, M. Kurdi and H. Heerklotz (2012). Classifying surfactants with respect to their effect on lipid membrane order, Biophys J. 102(3), 498-506.

[29] D.M. Livermore (1996). Are all beta-lactams created equal?, Scand. J. Infect. Dis. 101, 33-43. 
[30] P. Gilbert, D.J. Evans, E. Evans, I.G. Duguid and M.R. Brown (1991). Surface characteristics and adhesion of Escherichia coli and Staphylococcus epidermidis, J. Appl. Bacteriol. 71(1), 72-77.

[31] G. Eumkeb, S. Siriwong, S. Phitaktim, N. Rojtinnakorn and S. Sakdarat (2012). Synergistic activity and mode of action of flavonoids isolated from smaller galangal and amoxicillin combinations against amoxicillin-resistant Escherichia coli, J. Appl. Microbiol. 112(1), 55-64.

[32] G. Eumkeb, S. Siriwong and K. Thumanu (2012). Synergistic activity of luteolin and amoxicillin combination against amoxicillin-resistant Escherichia coli and mode of action, J. Photochem. Photobiol. B Biology. 117, 247-253.

[33] J.M. Bolla, S. Alibert-Franco, J. Handzlik, J. Chevalier, A. Mahamoud and G. Boyer (2011). Strategies for bypassing the membrane barrier in multidrug resistant Gram-negative bacteria, FEBS Lett. 585(11), 16821690.

[34] E.E. Tymczyszyn, M. del Rosario Diaz, A. Gomez-Zavaglia and E.A. Disalvo (2007). Volume recovery, surface properties and membrane integrity of Lactobacillus delbrueckii subsp. bulgaricus dehydrated in the presence of trehalose or sucrose, J. Appl. Microbiol. 103(6), 2410-2419.

[35] H.C. van der Mei, J. de Vries and H.J. Busscher (1993). Hydrophobic and electrostatic cell surface properties of thermophilic dairy streptococci, Appl. Environ. Microbiol. 59(12), 4305-4312.

[36] A. Borges, C. Ferreira, M.J. Saavedra and M. Simoes (2013). Antibacterial activity and mode of action of ferulic and gallic acids against pathogenic bacteria, Microb. Drug. Resist. 19(4), 256-265.

[37] V.K. Bajpai, A. Sharma and K.H. Baek (2013). Antibacterial mode of action of Cudrania tricuspidata fruit essential oil, affecting membrane permeability and surface characteristics of food-borne pathogens, Food Control. 32(2), 582-590.

[38] A. Sharma, V.K. Bajpai and K.H. Baek (2013). Determination of antibacterial mode of action of Allium sativum essential oil against foodborne pathogens using membrane permeability and surface characteristic parameters, J. Food. Safety. 33(2), 197-208.

[39] P. Souren, R.C. Dubey, D.K. Maheswari and K. S.C (2011). Trachyspermum ammi (L.) fruit essential oil influencing on membrane permeability and surface characteristics in inhibiting food-borne pathogens, Food Control. 22, 725-731.

[40] Y.S. Cho, J.J. Oh and K.H. Oh (2011). Synergistic anti-bacterial and proteomic effects of epigallocatechin gallate on clinical isolates of imipenem-resistant Klebsiella pneumoniae, Phytomedicine 18(11), 941-946.

[41] C. Fuqua and E.P. Greenberg (2002). Listening in on bacteria: Acyl-homoserine lactone signalling, Nat. Rev. Mol. Cell. Biol. 3, 685-695.

[42] S.J. Hagen (2016). The physical basis of bacterial quorum communication. Springer New York, New York.

[43] J.R. Edwards and M.J. Betts (2000). Carbapenems: the pinnacle of the $\beta$-lactam antibiotics or room for improvement?, J. Antimicrob. Chemother. 45(1), 1-4.

[44] G. Meletis, M. Exindari, N. Vavatsi, D. Sofianou and E. Diza (2012). Mechanisms responsible for the emergence of carbapenem resistance in Pseudomonas aeruginosa, Hippokratia 16(4), 303-307.

[45] Y.H. Li and X. Tian (2012). Quorum sensing and bacterial social interactions in biofilms, Sensors (Basel). 12(3), 2519-2538.

[46] C.F. Carson, B.J. Mee and T.V. Riley (2002). Mechanism of action of Melaleuca alternifolia (tea tree) oil on Staphylococcus aureus determined by time-kill, lysis, leakage, and salt tolerance assays and electron microscopy, Antimicrob. Agents. Chemother. 46(6), 1914-1920.

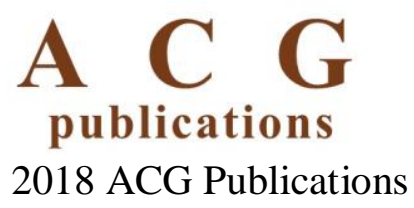

